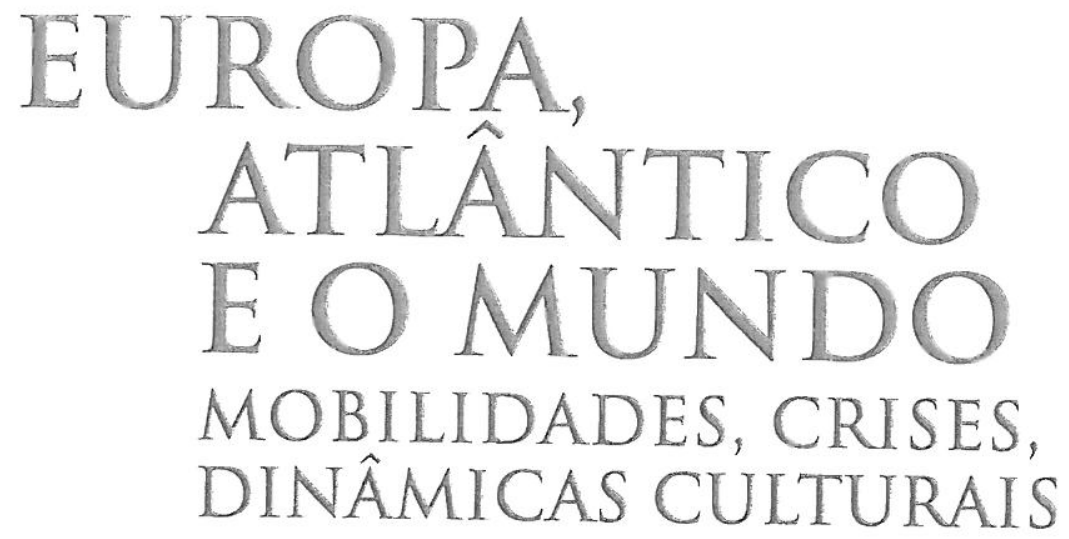

PENSAR COM

MARIA MANUELA TAVARES RIBEIRO

EUROPE, THE ATLANTIC AND THE WORLD MOBILLTT, CRISES, CULTURAL DYNAMICS

THINKING WITH MARIA MANUELA TAVARES RIBEIBO

COORDENAÇÃO

ISABEL MARIA FREITAS VALENTE 
Título / Tìle: Europa, Atlântico e o Mundo. Mobilidades, Crises, Dinâmicas Culturais. Pensar com Maria Manuela Tavares Ribeiro

Europe, the Atlantic and the World. Mobility, Crises, Cultural Dymanics.

Thinking with Moria Manuele Taveres Ribeiro

Coordenação / Edito: Isabel Maria Freitas Valente

Conselho Científico / Scientific board: Ariane Landuyt; Isabel Maria Freitas Valente; Giuliana Laschi; Luís Vieira de Andrade

Revisão / Review: Maria João Seabra

Paginação e Impressão / Book design and layoul: Pantone4

Edição / Eclition: Imprensa da Universidade de Coimbra

ISBN Impresso: 978-989-26-1335-2

ISBN Digital: 978-989-26-1336-9

Depósito legal: 424055/17

DOI: https://doi.org/10.14195/978-989-26-1336-9

Os dados e as opiniões inseridos na presente publicação sào da exclusiva responsabilidade dos seus autores.

Toda a reprodução desta obra, por fotocópia ou outro qualquer processo, sem a prévia autorização escrita do Editor, é ilícita e passível de procedimento judicial contra o infractor.

Alto Patrocinio

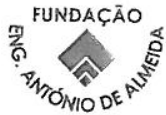

Apoios

2

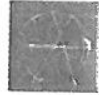

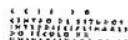
ชol.

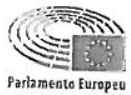




\title{
ROTAS DA CIÊNCIA E DA SAÚDE: INOVAÇÃO EM ANTIBIÓTICOS ENTRE A EUROPA E OS ESTADOS UNIDOS DA AMÉRICA \\ (1940-1960)
}

\author{
Victoria Bell \\ Professora Auxiliar Convidada da FFUC \\ Investigadora do CEIS20-UC \\ E-mail: victoriabell@ff.uc.pt \\ Ana Leonor Pereira \\ Professora Auxiliar da FLUC \\ Investigadora e co-coordenadora do GHSCT-CEIS20-UC \\ E-mail: aleop@ci.uc.pt \\ João Rui Pita \\ Professor Associado com Agregação da FFUC \\ Investigador e co-coordenador do GHSCT-CEIS20-UC \\ E-mail: jrpita@ci.uc.pt
}

\section{Resumo}

Neste estudo os autores apresentam um breve historial dos principais antibióticos entre os anos 40 e o início dos anos 60 do século XX remontando à penicilina dada a sua posição inaugural no processo de investigação dos antibióticos. Assim, torna-se claro como a pesquisa neste domínio iniciada na Grã-Bretanha, em plena Segunda Guerra Mundial, se transferiu para os Estados Unidos da América envolvendo a mobilidade de cientistas e em muitos casos a originalidade dos recursos naturais para a obtenção dos antibióticos. Os autores mostram, também, como a descoberta de uma vasta galeria de antibióticos depois da entrada da penicilina na 
terapêutica veio aumentar o arsenal medicamentoso e preencher lacunas existentes no tratamento das doenças infeciosas. Os efeitos nas dinâmicas e nas mobilidades demográficas, sociais e culturais desta complexa inovação científica, médica e farmacêutica continuaram a registar-se até hoje.

Palavras-chave: antibióticos; medicamentos; história das ciências farmacêuticas; século XX.

\section{Abstract}

In this study the authors present a brief history of the main antibiotics discovered between the 1940's and the 1960's. Penicillin is referred, by the authors, as the starting point for the discovery and development of other antibacterial products. It becomes clear how the research in this domain that began in England, during World War II, was transferred to the United States of America, involving the transference of knowledge and scientists and the screening of natural products to obtain new antibiotics. The authors also show how a new phase in antibacterial therapy, initiated by penicillin, resulted in profound changes in the assessment of infectious diseases and the way they were treated. The effect on demographic, social and cultural dynamics and mobility of this complex scientific, medical and pharmaceutical innovation remain visible today.

Keywords: Antibiotics; medicines; history of pharmacy; Twentieth century

A descoberta da penicilina em 1928 e a sua posterior transformação em medicamento nos anos 40 do século $\mathrm{XX}$ foi um longo processo com impacto decisivo na investigação de novas moléculas com propriedades antimicrobianas. A penicilina abriu as portas à antibioterapia e à descoberta de novos antibióticos ${ }^{1}$. Sublinha-se com frequência a se-

${ }^{1}$ Cf. TOMASZ, Alexander - «Lessons from the first antibiotic era". In Fighting infection in the 21st Century. Oxford: Blackwell Science Ltd, 2000, pp. 198-216. 
rendipidade $^{2}$ e o acaso ${ }^{3}$ como dois fatores decisivos na descoberta da penicilina por Alexander Fleming. Contudo, o mesmo não se pode dizer na descoberta de outros antibióticos. Com efeito, o investimento científico que foi feito na descoberta de outras moléculas com propriedades antimicrobianas resultou essencialmente de uma intensa investigação científica $^{4}$ que era, justamente, realizada com o objetivo explícito de alcançar novas substâncias antibióticas.

Depois da comprovação das propriedades terapêuticas da penicilina a indústria farmacêutica não hesitou em investir os seus meios económicos, humanos e científicos em projetos de screening que tinham por objetivo a pesquisa de substâncias com propriedades antibióticas ${ }^{5}$. O laboratório e a síntese química passavam a ser um outro caminho relativamente ao campo dos produtos naturais.

A publicação por Alexander Fleming (1881-1955), em 1929, do artigo "On the antibacterial action of cultures of a penicillium with special reference to their use in the isolation of B.influenzae", na prestigiada revista The British Journal of Experimental Pathology 6 marca um ponto de viragem no tratamento das doenças infeciosas. Com efeito, estava dado o primeiro passo na descoberta de um primeiro produto natural que podia eventualmente ter significativos efeitos benéficos no organismo humano no combate a infeções. A descoberta da penicilina por Alexander Fleming teve lugar em 1928. Fleming era microbiologista no Hospital de

2 Cf. LEDNICER, Daniel - New drug discovery and development. New Jersey: John Wiley \& Sons, Inc, 2007, p. 2.

3 Cf. WALLER, John - Fabulous science: fact and fiction in the history of scientific discovery. Oxford: Oxford University Press, 2004. p. 247.

${ }^{4}$ FLEMING, Alexander - «History and development of penicillin». In Penicillin: its practical application. London: Buttereworth \& Co., Ltd, 1946, pp. 1-23.

5 Cf. BUSH, K. - "Antibacterial drug discovery in the 21st century». In Clinical microbiology and infection: the official publication of the European Society of Clinical Microbiology and Infectious Diseases. V. 10, Suppl 4, 2004, pp. 10-17.

${ }^{6}$ FLEMING, Alexander - «On the antibacterial action of cultures of a penicillium with special reference to their use in the isolation of B.influenzae». In The British Journal of Experimental Pathology. V. 10, N. ${ }^{\circ} 3,1929$, pp. 226-236. 
St Mary, em Londres ${ }^{7}$. Em 1929 foi publicado o primeiro artigo referindo os efeitos benéficos anti-infeciosos da penicilina. No entanto, somente cerca de década e meia depois é que começaram a ser comercializados medicamentos contendo penicilina, na sequência de uma larga divulgação dos seus efeitos benéficos. Se Fleming e colaboradores tiveram um papel capital nos trabalhos iniciais de descoberta, caracterização do fármaco e colocação de hipóteses sobre as suas propriedades antimicrobianas, numa etapa posterior foram capitais os estudos da equipa liderada pelo investigador da Universidade de Oxford Howard Florey (1898-1968), em que Ernst Boris Chain (1906-1979) se revelou decisivo. Estes cientistas conseguiram obter o primeiro medicamento com penicilina. Com a sua perseverança foram ultrapassados obstáculos diversos como sejam a extração do produto, a sua purificação, a sua estabilidade, etc. ${ }^{8}$. Deve salientar-se que o contexto proporcionado pela Segunda Guerra Mundial não era propício à realização de estudos laboratoriais consistentes, seguros e economicamente viáveis pelo que a investigação sobre a penicilina inicialmente realizada na Grã-Bretanha foi levada para os Estados Unidos da América. Neste particular foi decisivo o papel de Howard Florey. Em 1939, Florey e Chain submeteram à Rockefeller Foundation de Nova Iorque uma candidatura a bolsa de investigação para se dedicarem a investigar as propriedades químicas e biológicas de substâncias antibacterianas produzidas por fungos e bactérias, entre as quais a penicilina, o que lhes foi concedido?. Estavam lançados os dados para que fossem realizados estudos muito aprofundados sobre

${ }^{7}$ No Hospital de St Mary funcionava uma Faculdade de Medicina, sendo um local de prestígio do ensino médico e de investigação científica.

${ }^{8}$ São variados os estudos no panorama internacional sobre aspetos diversos da história da penicilina, Veja-se sobre este assunto o estado da arte da tese de doutoramento de BELL, Victoria - Introdução dos antibióticos em Portugal: ciência, técnica e sociedade (anos 40 a 60 do século XX). Estudo de caso da penicilina. Coimbra: Universidade de Coimbra, 2014.

9 Cf. BUD, Robert - Penicillin Triumph and Tragedy. Oxford: Oxford University Press, 2007, p. 29; MAUROIS, André - The life of Sir Alexander Fleming. Oxford: The Alden Press, 1959, p. 162. 
a penicilina até se conseguir obter com ela um medicamento. Foi um processo que envolveu muitos cientistas e diversas instituições. A cada passo dado os resultados iam-se consolidando. Em Agosto de 1940 os investigadores de Oxford publicaram na revista The Lancet, um artigo intitulado "Penicillin as a Chemotherapeutic Agent"10. Neste estudo, autêntico relatório preliminar sobre as pesquisas realizadas sobre as propriedades químicas, farmacológicas e quimioterapêuticas da penicilina, são mencionados os trabalhos anteriores publicados por Fleming.

A primeira administração de penicilina em doente nos Estados Unidos da América verificou-se em 1942, depois de aturadas investigações, publicações, investimentos científicos e económicos e no contexto de uma partilha muito profícua de interesses entre cientistas de equipas dos Estados Unidos da América e da Grã-Bretanha. A Segunda Guerra Mundial veio funcionar como catalisador dos trabalhos de investigação em curso sobre a penicilina e sobre a sua utilização fora da comunidade científica. A penicilina deveria ser entendida como uma arma contra as doenças infeciosas e capaz de rapidamente recuperar militares infetados. A sua utilização em massa nas tropas aliadas foi uma realidade. O circuito da penicilina estava a abrir-se. Os anos de 1943 e de 1944 marcam justamente a saída da penicilina para fora do circuito científico. Chegou a Portugal em 1944 através da Cruz Vermelha Portuguesa e proveniente da congénere americana ${ }^{11}$.

Logo após a descoberta da penicilina e depois de terem sido confirmadas as suas propriedades antimicrobianas, inigualadas por qualquer medicamento, verificou-se que este fármaco não se revelava eficiente no

10 CHAIN, E. B. et al. - «Penicillin as a chemotherapeutic agent». In The Lancet. V. 236, N. ${ }^{\circ}$ 6104, 1940, pp. 226-228.

11 Sobre este assunto e, em particular a entrada em Portugal, veja-se: BELL, Victoria - Introdução dos antibióticos em Portugal: ciência, técnica e sociedade (anos 40 a 60 do século $X X$ ). Estudo de caso da penicilina. Coimbra: Universidade de Coimbra, 2014. Ver também sobre a cooperação entre o Brasil, os Estados Unidos da América e Portugal na receção da penicilina: BELL, Victoria; PEREIRA, Ana Leonor; PITA, João Rui — "The reception of penicillin in Portugal during the World War II: coperation with Brazil and United States of America». In Debater a Europa. V. 13, 2015, pp. 143-157. 
tratamento da tuberculose ${ }^{12}$. Ainda em plena Segunda Guerra Mundial as investigações sobre antibióticos continuaram a avançar intensamente na esperança de se conseguir arranjar uma galeria diversificada de medicamentos antimicrobianos capazes de combater intensamente a variedade de infeções então conhecidas.

A tuberculose, tal como outras doenças infeciosas, constituía um grave problema de saúde privada e pública. Tentativas realizadas com outros fármacos em contexto sanatorial ou noutro contexto eram as possíveis mas não as desejáveis para o tratamento da doença.

A descoberta do primeiro antibiótico para o tratamento da tuberculose está intimamente associada ao nome de Selman A. Waksman (1888-1973) um bioquímico norte-americano (ucraniano de nascimento), de origem judaica, investigador da Universidade de Rutgers, nos Estados Unidos da América. A busca incessante para conseguir um medicamento eficaz no tratamento da tuberculose estimulava Waksman e sua equipa a realizarem estudos sobre o assunto. A produtividade dessas investigações ficou plasmada em estudos científicos. Assim, em 1944 a revista Proceedings of the Society for Experimental Biology and Medicine publicou o artigo intitulado "Streptomycin, a Substance Exhibiting Antibiotic Activity Against Gram-Positive and Gram-Negative Bacteria"13 da autoria de Albert Schatz, Elizabeth Bugle e Selman A. Waksman, investigadores do Department of Biochemistry and Microbiology da Rutgers University, nos Estados Unidos da América. Neste artigo os autores descreveram o isolamento, a partir de duas estirpes de actinomicetes, de uma substância com propriedades antibióticas. Esta substância foi denominada estreptomicina. No mesmo estudo os autores abordaram a sua ação farmacoterapêutica e as suas propriedades físico-químicas. Ainda no

\footnotetext{
12 LAWN, Stephen D.; ZUMLA, Alimuddin I. - «Tuberculosis». In The Lancet. V. 378, $\mathrm{N}^{\circ}$ 9785, 2011, pp.57-72

13 SCHATZ, A.; BUGLE, E.; WAKSMAN, S. A. - «Streptomycin, a Substance Exhibiting Antibiotic Activity Against Gram-Positive and Gram-Negative Bacteria». In Proceedings of the Society for Experimental Biology and Medicine. V. 55, 1944, pp. 66-69.
} 
mesmo ano, no mês de dezembro, W. H. Feldman e H. C. Hinshaw, investigadores da Mayo Clinic, também nos Estados Unidos da América publicaram o estudo intitulado "Effects of Streptomycin on Experimental Tuberculosis in Guinea Pigs: A Preliminary Report”14. Neste artigo os autores descreveram os estudos realizados para determinar a eficácia da estreptomicina no tratamento da tuberculose em animais. Os resultados a que estes investigadores chegaram foram muito positivos e animaram Feldman e Hinshaw a prosseguirem o seu estudo sobre a ação do fármaco no Mycobacterium tuberculosis. O objetivo era realizar os primeiros ensaios clínicos em seres humanos. Estava na mente dos investigadores conseguir um medicamento eficaz para o combate à tuberculose que nos anos 40 do século XX continuava sem solução farmacológica.

Logo no ano seguinte, em 1945, no artigo "Streptomycin in Treatment of Clinical Tuberculosis: A Preliminary Report" 15 publicado em Proceedings of the Staff Meetings of the Mayo Clinic os autores, H. C. Hinshaw e W. H. Feldman, descreveram as suas observações clínicas. A eficácia demonstrada pela estreptomicina no tratamento da tuberculose era evidente o que levou à realização de ensaios clínicos mais consistentes e abrangentes de modo a determinar outras aplicações terapêuticas do fármaco ${ }^{16}$. Deste modo, a estreptomicina alterava radicalmente o tratamento da tuberculose ${ }^{17}$. Pela primeira vez havia um fármaco com capacidade para tratar a doença e assim passou a contribuir drasticamen-

\footnotetext{
14 FELDMAN, W. H.; HINSHAW, H. C. - "Effects of Streptomycin on Experimental Tuberculosis in Guinea Pigs: A Preliminary Report». In Proceedings of the Staff Meetings of the Mayo Clinic. V. 19, 1944, pp. 593-599.

15 HINSHAW, H. C.; FELDMAN, W. H. - "Streptomycin in Treatment of Clinical Tuberculosis: A Preliminary Report». In Proceedings of the Staff Meetings of the Mayo Clinic. V. 20, 1945, pp. 313-318.

16 KEEFER, Chester S. et al. - "Streptomycin in the treatment of infections - A Report of One Thousand Cases». In The Journal of the American Medical Association. V. 132, 1946, pp. 4-10; pp. 70-77.

17 BRUMFITT, W.; HAMILTON-MILLER, J. M. T. — "The changing face of chemotherapy». In Postgraduate Medical Journal. V. 64, N. ${ }^{\circ} 753,1988$, pp. 552-558.
} 
te para a diminuição da mortalidade associada à doença ${ }^{18}$. Deve salientar-se que em 1952 foi atribuído a Selman Waksman o Prémio Nobel de Medicina ou Fisiologia pela descoberta da estreptomicina, considerado o primeiro antibiótico eficaz no tratamento da tuberculose.

Também em 1943 um grupo de investigadores da Universidade de Columbia em Nova Iorque, Estados Unidos da América, descreveu o isolamento de uma substância antibiótica, a bacitracina, a partir de culturas de Bacillus subtilis ${ }^{19}$. O resultado do seu trabalho de investigação foi publicado em 1945. Apesar de a bacitracina ter revelado ser um promissor agente antibacteriano a elevada toxicidade renal demonstrada limitou a sua aplicabilidade terapêutica ${ }^{20}$. Esta toxicidade renal associada à administração injetável limitou o seu uso, sobretudo, ao tratamento de infeções oculares, do aparelho auditivo externo, algumas infeções urinárias e pulmonares.

A investigação realizada pela indústria farmacêutica no sentido de se descobrirem novas substâncias antibióticas intensificava-se de modo significativo. Havia a consciência de que os resultados alcançados com a penicilina e a estreptomicina abriam as portas à descoberta de novos fármacos antibióticos. O combate consistente às doenças infeciosas havia começado de um modo sólido. As pesquisas que estavam a ser

\footnotetext{
18 A importância da estreptomicina no tratamento da tuberculose sobressai na tese de doutoramento de VIEIRA, Ismael Cerqueira - Conbecer, tratar e combater a 'peste branca'. A tisiologia e a luta contra a tuberculose em Portugal (1853-1975). Porto: Faculdade de Letras - Universidade do Porto, 2012, p. 268. Neste valioso e volumoso estudo sobre a tuberculose em Portugal os antibióticos não constituíram uma preocupação principal. Contudo, a importância do antibiótico é sublinhada. A par da estreptomicina outros fármacos foram também utilizados após a sua descoberta ou síntese. Foi o caso da isoniazida (1952), da rifampicina (1965), do etambutol (síntese em 1960 e utilizaçãoo em 1968) e da pirazinamida (sintetizada em 1936 e utilizada em 1970). Sobre este assunto ver: SOUZA, Marcus Vinícius Nora de; VASCONCELOS, Thatyana Rocha Alves - "Fármacos no combate à tuberculose: passado, presente e futuro". In Quimica Nova. V. 28, N. ${ }^{4}$, 2005, p. 679.

19 JOHNSON, Balbina A.; ANKER, Herbert; MELENEY, Frank L. - "Bacitracin: A new antibiotic produced by a member of the B. subtilis group". In Science. V. 102, N. ${ }^{\circ} 2650$, 1945, pp. 376-377.

20 NEWTON, G. G. F. et al. - "Some observations on the biological properties of bacitracins A, B, and C'. In British Journal of Pharmacology and Chemotherapy. V. 6, N. ${ }^{\circ}$ 3, 1951, pp. 417-429.
} 
feitas eram muitas e dispersas por muitos laboratórios de investigação. Por isso, muito naturalmente, em 1947, surge a descoberta de um novo antibiótico. Com efeito, a partir de uma amostra de solo proveniente da Venezuela foi isolada uma nova substância com propriedades antibióticas. A essa substância foi dado o nome de cloromicetina. A sua descoberta e identificação foi feita por uma equipa de químicos do laboratório farmacêutico Parke-Davis and Company, liderada por John Ehrlich. Esta indústria farmacêutica, fundada nos anos sessenta do século XIX em Detroit, nos Estados Unidos da América, era uma instituição com um significativo investimento na investigação científica. A descoberta da cloromicetina foi divulgada na revista Science no artigo "Chloromycetin, a new antibiotic from a soil actinomycete" 21 . Como se vê, o próprio título do artigo é esclarecedor ao referir um "novo antibiótico". Na verdade, estava-se na presença de um processo de construção do novo na terapêutica medicamentosa. Não se tratava apenas de uma inovação que tinha a ver com alguma alteração de um produto pré-existente. Tratava-se da descoberta de um fármaco inovador que proporcionaria um novo medicamento que vinha preencher uma lacuna terapêutica. A cloromicetina veio posteriormente a ser produzida por síntese química tornando-se no primeiro antibiótico obtido por esta via o que simbolicamente apresenta um enorme interesse. Foi atribuída a denominação de cloranfenicol ao antibiótico obtido por síntese química ${ }^{22}$. Deve dizer-se que esta obtenção por síntese química reforçava ainda mais a originalidade do novo fármaco. A cloromicetina demonstrou grande eficácia no tratamento da febre tifoide. Deve lembrar-se que a febre tifoide constituía um enorme problema de saúde pública sem terapêutica medicamentosa curativa ou preventiva eficaz. O facto de ser obtida por síntese química era uma distinção relativamente aos restantes antibióticos. Por esse facto, cerca de

${ }^{21}$ EHRLICH, John et al. - "Chloromycetin, a new antibiotic from a soil actinomycete». In Science. V. 106, N. ${ }^{\circ} 2757,1947$, p. 417.

22 CARVALHO, Luís da Silva - "A cloromicetina». In Notícias Farmacêuticas. V. 15, N. ${ }^{\circ}$ 7-8, 1949, pp. 312-339. 
três anos após o seu lançamento no circuito comercial, em 1949, as suas vendas atingiram 120 milhões de dólares contribuindo para o sucesso da empresa Parke-Davis and Company ${ }^{23}$. Em 1952 começaram a surgir relatos de casos de anemia aplástica após a administração do antibiótico ${ }^{24}$. A incidência e a gravidade deste efeito adverso levou a que a FDA (Food and Drug Administration ${ }^{25}$ ) em conjunto com a indústria farmacêutica Parke-Davis realizassem um inquérito em hospitais e clínicas para averiguar a extensão do problema ${ }^{26}$. Em 1954 o Council on Pharmacy and Chemistry aconselhou que a utilização do antibiótico ficasse restringida ao tratamento da febre tifoide e de patologias resistentes a outros antibióticos. As reações adversas relatadas e que constituíam um problema na utilização do antibiótico contribuíram para a diminuição das vendas de medicamentos com aquele fármaco, deixando este de assumir uma posição relevante na indústria. O declínio nas vendas de cloromicetina terá sido um dos fatores responsáveis pela fusão da Parke-Davis com a Warner-Lambert, em $1974^{27}$.

Em 1948, como resultado de trabalhos de investigação realizados pelo laboratório farmacêutico Lederle, foi anunciada a descoberta de outro antibiótico, a aureomicina. Benjamin Minge Duggar (1872-1956), botânico, professor de botânica da Universidade de Missouri, Universidade de Washington e do Jardim Botânico de Missouri, nos Estados Unidos da América, foi colaborador da referida indústria farmacêutica e nessa condição interessou-se particularmente pela pesquisa de substâncias com

23 Cf. HOEFLE, Milton L. - "The early history of Parke-Davis and Compant». In Bulletin of the History of Chemistry. V. 25, N. ${ }^{\circ} 1,2000$, pp. 28-34.

24 WINTERNITZ, C. - «Fatal aplastic anemia following chloramphenicol (chloromycetin) therapy». In California medicine. V. 77, N. ${ }^{\circ}$ 5, 1952, pp. 335-339.

25 A FDA é o órgão governamental dos Estados Unidos da América que tutela o controlo de alimentos e suplementos alimentares, bem como medicamentos, cosméticos, derivados do sangue e materiais biológicos e ainda dispositivos médicos.

26 RENICHNICK, Pierre - «Os acidentes provocados pelos antibióticos». In Jornal do Médico. V. 21, N. ${ }^{\circ}$ 524, 1953, pp. 318-326.

27 Cf. HOEFLE, Milton L. - "The early history of Parke-Davis and Compant». In Bulletin of the History of Chemistry. V. 25, N. 1, 2000, pp. 28-34. 
propriedades antibacterianas. No artigo "Aureomycin: a product of the continuing search for new antibiotics" 28 referia, em 1948, o isolamento da aureomicina a partir de uma estirpe de Streptomyces, Streptomyces aureofaciens. No estudo o autor descreveu a ação da substância na inibição de diversas estirpes de bactérias e referiu que estavam a ser realizados ensaios clínicos para determinar a sua aplicação terapêutica. Um destes primeiros ensaios clínicos decorreu no Boston City Hospital, nos Estados Unidos da América, e constou do tratamento de 100 doentes com aureomicina por via oral. O resultado dos trabalhos foi publicado pelos autores Harvey Shields Collins, Thomas Fite Paine e Maxwell Finland, na revista norte-americana Annals of the New York Academy of Sciences no artigo intitulado "Clinical studies with aureomycin"29. Os mesmos autores publicaram também o resultado da pesquisa na revista The Journal of the American Medical Association no artigo "Aureomycin, a new antibiotic. Results of Laboratory Studies and of Clinical Use in 100 Cases of Bacterial Infections"30. O nome de aureomicina provem justamente do aspeto dourado que apresentava o fungo com propriedades antibióticas. Verificou-se que a aureomicina era um fungo muito frequente nos terrenos que estão muito impregnados com dejetos de animais, tendo-se constatado ser comum em terrenos onde pastava frequentemente gado bovino ${ }^{31}$. Mais tarde, a aureomicina veio a designar-se

\footnotetext{
28 DUGGAR, B. M. - «Aureomycin: a product of the continuing search for new antibiotics". In Annals of the New York Academy of Sciencesmy of Sciences. V. 51, N. ${ }^{\circ} 2,1948$, pp. 177-181.

29 COLLINS, Harvey Shields; PAINE, Thomas Fite; FINLAND, Maxwell - "Clinical studies with aureomycin”. In Annals of the New York Academy of Sciences.V. 51, 1948, pp. 231-240.

30 FINLAND, Maxwell; COLLINS, Harvey Shields; PAINE, Thomas Fite - «Aureomycin, a new antibiotic. Results of Laboratory Studies and of Clinical Use in 100 Cases of Bacterial Infections". In The Journal of the American Medical Association. V. 138, N. ${ }^{\circ} 13$, 1948, pp. 946-949.

31 Cf. ANDRADE, Carlos Henrique Vianna de - História ilustrada da medicina na Antiguidade. São Paulo: Barauna, 2012, p. 139.
} 
por clorotetraciclina. Deve lembrar-se que a aureomicina é considerada como a primeira tetraciclina a ter sido descoberta.

Recorde-se que tanto a aureomicina como a cloromicetina apresentavam na época a vantagem de serem bem absorvidos por via oral. Deste modo simplificava-se a administração do medicamento e estava igualmente favorecida e facilitada a adesão à terapêutica por parte dos doentes. Estes novos antibióticos também dispunham de um espetro de ação mais alargado do que a penicilina permitindo a sua utilização em patologias resistentes àquele antibiótico o que lhes conferia claras vantagens terapêuticas. Apesar dos (aparentes) benefícios demonstrados inicialmente por estes antibióticos relativamente à penicilina, a toxicidade por eles revelada pela utilização alargada em ambulatório limitou substancialmente o seu emprego na terapêutica, ao invés da penicilina que, pela inocuidade demonstrada, continuou a ser amplamente utilizada no combate às doenças infeciosas.

Após a descoberta e consolidação terapêutica da penicilina, a utilização da estreptomicina e da cloromicetina constitui um outro marco relevante na história dos antibióticos. Este marco nas rotas da ciência e da saúde significa o preenchimento de lacunas terapêuticas, o enriquecimento do arsenal terapêutico, o incentivo à procura de novas substâncias com propriedades antimicrobianas e, obviamente, significa o alargamento dos efeitos benéficos no plano da esperança de vida, da qualidade de vida e da saúde de muitas populações. Após as referidas descobertas, muitas outras substâncias foram isoladas do solo; no entanto, muito poucas demonstraram aplicabilidade terapêutica devido à sua elevada toxicidade.

Selman A. Waksman dedicou-se afincadamente ao estudo de microrganismos provenientes do solo com capacidade de produzirem substâncias inibidoras que atuassem nas bactérias resistentes à estreptomicina. Em 1949 isolou, a partir do Streptomyces fradiae, a neomicina. O investigador descreveu a descoberta e as propriedades do antibiótico no artigo "Neomycin, a new antibiotic active against streptomycin-resistant bac- 
teria, including tuberculosis organisms" ${ }^{\prime 2}$ publicado na revista Science. Estudos preliminares indicavam que o antibiótico poderia ter grande utilidade terapêutica ${ }^{33}$. No entanto, a sua elevada toxicidade limitou a sua utilização à aplicação tópica.

Em 1950 uma equipa de investigadores do laboratório farmacêutico Pfizer isolou, a partir do Streptomyces rimosus, a terramicina, uma substância que revelou possuir, in vitro, notáveis propriedades antibióticas e uma baixa toxicidade em estudos com animais ${ }^{34}$. Estudos clínicos posteriores determinaram que a terramicina (também denominada oxitetraciclina) era bem tolerada, que poderia ser administrada por via oral e que os efeitos adversos reportados não eram graves ${ }^{35}$. No entanto, o aparecimento de resistências bacterianas limitou a sua utilização terapêutica ${ }^{36}$.

As propriedades de um conjunto de substâncias antibióticas, isoladas a partir de uma espécie de Cephalosporium, foram descritas em 1951 por uma equipa de investigadores do Sir William Dunn School of Pathology da Universidade de Oxford da qual fazia parte Edward Penley Abraham, um dos principais investigadores da equipa de Howard Florey. Recorde-se que Florey foi um dos investigadores participantes na investigação sobre a penicilina e sobre a sua transformação em medicamento. Trata-se, na rota das descobertas de antibióticos, entre os anos 40 e o início dos anos 50, do retorno à Grã-Bretanha, país de origem dos antibióticos com a descoberta da penicilina. No artigo "Isolation of Antibiotics from

\footnotetext{
32 WAKSMAN, Selman A.; LECHEVALIER, Hubert A. - "Neomycin, a new antibiotic active against streptomycin-resistant bacteria, including tuberculosis organisms». In Science. V. 109, N. $^{\circ} 2830,1949$, pp. 305-304.

33 "A neomicina - um novo antibiótico activo contra bactérias resistentes à estreptomicina incluindo o microrganismo da tuberculose». In Jornal do Médico. V. 16, N. ${ }^{\circ} 394$, 1950 , p. 233.

34 FINLAY, A. C. et al. - "Terramycin, a new antibiotic». In Science. N. ${ }^{\circ} 111,1950$, p. 85.

35 "A terramicina no tratamento da pneumonia pneumocócica e da pneumonia atípica primária». In Jornal do Médico. V. 16, N. ${ }^{\circ} 409$, 1950, p. 800.

36 FUSILLO, Matthew H. et al. - "Phage typing of antibiotic-resistant staphylococci». In American Journal of Public Health. V. 44, N. ${ }^{\circ} 3$, 1954, pp. 317-322.
} 
a Species of Cephalosporium. Cephalosporins P1, P2, P3, P4 and P5"37 publicado na revista Biochemical Journal, os autores descreveram os métodos de isolamento de uma nova substância antibiótica que denominaram cefalosporina $\mathrm{P}$, retrataram as suas propriedades físico-químicas e mencionaram também que além da cefalosporina $\mathrm{P}$, nos fluídos da cultura de Cephalosporium foi identificada outra substância antibiótica, a cefalosporina $\mathrm{N}$, com atividade antibacteriana e propriedades químicas distintas da cefalosporina P. Determinaram que a cefalosporina $\mathrm{P}$ era na realidade um conjunto de cinco moléculas (cefalosporina P1, P2, P3, P4 e P5) das quais uma, P1, estava presente em maiores quantidades. A cefalosporina P1 revelou possuir atividade contra estafilococos. No entanto, os autores indicaram que estes microrganismos adquiriam resistência ao antibiótico quando cultivados na sua presença. As propriedades biológicas da cefalosporina P1 também foram retratadas por outro grupo de investigadores do Sir William Dunn School of Pathology da Universidade de Oxford. Howard Florey e colaboradores publicaram, ainda em 1951, o artigo "Some biological properties of cephalosporin P1"38 onde descreveram os trabalhos realizados para determinar a atividade antibacteriana da cefalosporina P1. Através da sua investigação verificaram que o antibiótico era bem absorvido quando administrado por via oral e que a sua toxicidade era inferior à apresentada pela aureomicina e terramicina em estudos animais.

As condições de produção do antibiótico também foram objeto de estudo por um terceiro grupo de investigadores da mesma instituição. A investigação, realizada em conjunto com membros do Antibiotics Research Station do Medical Research Council em Clevedon, resultou na publicação do arti-

37 BURTON, H. S.; ABRAHAM, E. P. - "Isolation of Antibiotics from a Species of Cephalosporium. Cephalosporins P1, P2, P3, P4 and P5". In Biochemical Journal. V. 50, N. ${ }^{\circ}$ 2, 1951, pp. 168-174.

38 RITCHIE, A. C.; SMITH, N.; FLOREY, H. W. - «Some biological properties of cephalosporin P1". In British Journal of Pharmacology. V. 6, N. ${ }^{\circ} 3$, 1951, pp. 430-444. 
go "Antibiotic production by a species of Cephalosporium"39 onde são descritos os métodos de produção do antibiótico, com referência aos meios de cultura empregues e às técnicas utilizadas para aumentar a produtividade.

Após estes estudos iniciais, Edward Abraham continuou a investigar as substâncias antibióticas produzidas por culturas de Cephalosporium. Em 1954, juntamente com G. G. F. Newton, publicou dois artigos no Biochemical Journal onde retratou a purificação e as propriedades biológicas da cefalosporina $\mathrm{N}^{40}$ e a sua estrutura química ${ }^{41}$ e em 1955 identificou, com o mesmo coautor, cefalosporina $\mathrm{C}^{42}$ e descreveu o isolamento da mesma ${ }^{43}$. Em 1961, Edward Abraham e colaboradores identificaram o núcleo da cefalosporina C (ácido 7-aminocefalosporânico) ${ }^{44}$ que constituiu o ponto de partida para o início da produção por síntese química das cefalosporinas ${ }^{45}$. As disputas travadas entre os EUA e a Grã-Bretanha em relação ao registo de patentes da penicilina motivaram Edward Abraham a registar patentes de todas as suas descobertas científicas associadas às cefalosporinas ${ }^{46}$.

39 CRAWFORD, Kathleen et al. - "Antibiotic production by a species of Cephalosporium». In Journal of General Microbiology. V. 6, N. ${ }^{\circ}$ 1-2, 1952, pp. 47-59.

40 ABRAHAM, E. P.; NEWTON, G. G. F.; HALE, C. W. - «Purification and some properties of cephalosporin N, a new penicillin”. In Biochemical Journal. V. 58, N. ${ }^{\circ} 1,1954$, pp. 94-102.

41 NEWTON, G. G. F.; ABRAHAM, E. P. - «Degradation, structure and some derivatives of cephalosporin N». In Biochemical Journal. V. 58, N. ${ }^{\circ}$ 1, 1954, pp. 103-111.

42 NEWTON, G. G. F.; ABRAHAM, E. P. - «Cephalosporin C, a new antibiotic containing sulphur and D- $\alpha$-aminoadipic acid». In Nature. V. 175, N. ${ }^{\circ} 4456,1955$, p. 548.

43 NEWTON, G. G. F.; ABRAHAM, E. P. - «Isolation of cephalosporin C, a penicillin-like antibiotic containing D-alpha-aminoadipic acid». In Biochemical Journal. V. 62, N. ${ }^{\circ}$ 4, 1956, pp. 651-658.

${ }^{44}$ LODER, Bronwen; NEWTON, G. G. F.; ABRAHAM, E. P. - «The cephalosporin C nucleus (7-aminocephalosporanic acid) and some of its derivatives". In Biochemical Journal. V. 79, N. ${ }^{\circ} 4,1961$, pp. 408-416.

45 Cf. BRUMFITT, W.; HAMILTON-MILLER, J. M. T. - «The changing face of chemotherapy". In Postgraduate Medical Journal. V. 64, N. ${ }^{\circ} 753,1988$, pp. 552-558.

${ }^{46}$ LAX, Eric - The Mold in Dr. Florey's Coat. The story of the penicillin miracle. New York: Owl Books, 2005, pp. 251-252. 
A eritromicina isolada a partir do Streptomyces erytheus foi descrita por uma equipa de investigadores do laboratório farmacêutico Eli Lilly ${ }^{47}$. Os trabalhos da equipa chefiada por J. M. McGuire foram publicados em junho de 1952 na revista Suíça Schweizerische Medizinische Wochenschrift $^{48}$ e o seu efeito terapêutico foi confirmado em julho do mesmo ano por um grupo de investigadores da Mayo Clinic. No artigo "Some laboratory and clinical observations on a new antibiotic, erythromycin (ilotycin)" 49 os autores referem que o antibiótico demonstrou uma boa absorção após administração oral mas que em doses elevadas provocava irritação gastrointestinal. Mencionam que trabalhos efetuados apontam para uma boa ação do antibiótico numa grande variedade de infeções mas que serão necessários mais estudos para comprovar a sua eficácia, o que está em sintonia com o objetivo final da investigação que é proporcionar os melhores cuidados terapêuticos aos pacientes em nome dum bem supremo que é a sua saúde e a saúde pública.

\section{Considerações finais}

Com a introdução dos antibióticos na terapêutica pensou-se que as doenças infeciosas tinham chegado ao seu termo. Isto é: a solução terapêutica medicamentosa estava conseguida com êxito e, portanto, este problema estaria totalmente resolvido. Após as descobertas dos primeiros antibióticos gerou-se a ideia de que as doenças infeciosas com grandes taxas de mortalidade fariam parte do passado. Contudo, a euforia em torno da antibioterapia não terá valorizado o poder de adaptação que

47 A Eli Lilly é uma multinacional farmacêutica fundada nos Estados Unidos da América em 1876 que investiu na investigação científica e produção de medicamentos de grande importância e significado como foi o caso dos antibióticos, mas também, entre outros, insulina, vacinas contra a poliomielite e mais perto de nós o célebre Prozac.

48 MCGUIRE, J. M. et al. - "Ilotycin, a new antibiotic». In Schweizerische Medizinische Wochenschrift. V. 82, N. ${ }^{\circ} 41,1952$, pp. 1064-1065.

49 HEILMAN, F. R. et al. - "Some laboratory and clinical observations on a new antibiotic, erythromycin (ilotycin)". In Proceedings of the Staff Meetings of the Mayo Clinic. V. 27 , N. ${ }^{\circ} 15,1952$, pp. $285-304$. 
os micro-organismos, as bactérias, iam revelando ${ }^{50}$. A breve trecho os fenómenos de resistência aos antibióticos começaram a surgir. Foi notável o esforço despendido na investigação sobre antibióticos inicialmente na Grã-Bretanha pela mão de Alexander Fleming e depois por Howard Florey e Ernst Boris Chain e outros cientistas das equipas que conseguiram transformar em medicamento a penicilina e produzi-la à escala industrial. Se a génese da antibioterapia é na Grã-Bretanha, também é certo que os trabalhos realizados posteriormente na própria penicilina e depois conducentes à descoberta de outros antibióticos foram transferidos para os Estados Unidos da América. Esta rota compreende-se pois os recursos humanos e materiais dos EUA comparados com os de uma Europa abalada pela Segunda Guerra Mundial eram muito superiores.

Nesta obra intitulada "Europa, Atlântico, Mundo. Mobilidades, Crises, Dinâmicas Culturais" a descoberta dos primeiros antibióticos desde a penicilina às cefalosporinas constitui um exemplo significativo de mobilidade científica entre a Europa e os Estados Unidos da América com impacto planetário. Constitui também um modelo de produção e circulação de medicamentos com elevado nível de impacto nas dinâmicas sociais e culturais, desde logo por se afirmarem no tratamento de doenças que não tinham cura, diminuindo a taxa de mortalidade, aumentando a esperança de vida, melhorando a qualidade de vida a todos os níveis da existência desde o privado ao domínio público.

\section{Referências bibliográficas}

ABRAHAM, E. P.; NEWTON, G. G. F.; HALE, C. W. - «Purification and some properties of cephalosporin N, a new penicillin». In Biochemical Journal. V. 58, N. ${ }^{\circ} 1,1954$, pp. 94-102.

\footnotetext{
50 Cf. DAVIES, Julian - «Microbes have the last word». In European Molecular Biology
} Organazation Reports. V. 8, N. ${ }^{\circ}$ 7, 2007, pp. 616-621. 
ANDRADE, Carlos Henrique Vianna de - História ilustrada da medicina na Antiguidade. São Paulo: Barauna, 2012, p. 139.

BELL, Victoria - Introdução dos antibióticos em Portugal: ciência, técnica e sociedade (anos 40 a 60 do século XX). Estudo de caso da penicilina. Coimbra: Universidade de Coimbra, 2014.

BELL, Victoria; PEREIRA, Ana Leonor; PITA, João Rui - «The reception of penicillin in Portugal during the World War II: coperation with Brazil and United States of America». In Debater a Europa. V. 13, 2015, pp. 143-157.

BRUMFITT, W.; HAMILTON-MILLER, J. M. T. - «The changing face of chemotherapy». In Postgraduate Medical Journal. V. 64, N. ${ }^{\circ} 753,1988$, pp. 552-558.

BUD, Robert - Penicillin Triumph and Tragedy. Oxford: Oxford University Press, 2007, p. 29.

BURTON, H. S.; ABRAHAM, E. P. - «Isolation of Antibiotics from a Species of Cephalosporium. Cephalosporins P1, P2, P3, P4 and P5». In Biochemical Journal. V. 50, N. ${ }^{\circ}$ 2, 1951, pp. 168-174.

BUSH, K. - "Antibacterial drug discovery in the 21st century». In Clinical microbiology and infection : the official publication of the European Society of Clinical Microbiology and Infectious Diseases. V. 10, Suppl 4, 2004, pp. 10-17.

CARVALHO, Luís da Silva - "A cloromicetina». In Notícias Farmacêuticas. V. 15, N. ${ }^{\text {7 }}$-8, 1949, pp. 312-339.

CHAIN, E. B. et al. - «Penicillin as a chemotherapeutic agent». In The Lancet. 236, 6104 (1940), pp. 226-228.

COLLINS, Harvey Shields; PAINE, Thomas Fite; FINLAND, Maxwell - "Clinical studies with aureomycin". In Annals of the New York Academy of Sciences. V. 51, 1948, pp. 231-240.

CRAWFORD, Kathleen et al. - "Antibiotic production by a species of Cephalosporium». In Journal of General Microbiology. V. 6, N. ${ }^{\circ}$ 1-2, 1952 , pp. 47-59.

DAVIES, Julian - «Microbes have the last word». In European Molecular Biology Organazation Reports. V. 8, N. ${ }^{\circ}$ 7, 2007, pp. 616-621.

DUGGAR, B. M. - "Aureomycin: a product of the continuing search for new antibiotics». In Annals of the New York Academy of Sciencesmy of Sciences. V. 51, N. ${ }^{\circ} 2,1948$, pp. 177-181.

EHRLICH, John et al. - "Chloromycetin, a new antibiotic from a soil actinomycete». In Science. V. 106, N. ${ }^{\circ} 2757,1947$, p. 417.

FELDMAN, W. H.; HINSHAW, H. C. - «Effects of Streptomycin on Experimental Tuberculosis in Guinea Pigs: A Preliminary Report”. In Proceedings of the Staff Meetings of the Mayo Clinic. V. 19, 1944, pp. 593-599. 
FINLAND, Maxwell; COLLINS, Harvey Shields; PAINE, Thomas Fite - "Aureomycin, a new antibiotic. Results of Laboratory Studies and of Clinical Use in 100 Cases of Bacterial Infections». In The Journal of the American Medical Association. V. 138, N. $^{\circ} 13,1948$, pp. 946-949.

FINLAY, A. C. et al. - "Terramycin, a new antibiotic». In Science. N. ${ }^{\circ} 111,1950$, p. 85.

FLEMING, Alexander - «History and development of penicillin». In Penicillin: its practical application. London: Buttereworth \& Co., Ltd, 1946, pp. 1-23.

FLEMING, Alexander - «On the antibacterial action of cultures of a penicillium with special reference to their use in the isolation of B.influenzae». In The British Journal of Experimental Pathology. V. 10, N. ${ }^{\circ} 3$, 1929, pp. 226-236.

FUSILLO, Matthew H. et al. - «Phage typing of antibiotic-resistant staphylococci». In American Journal of Public Health. V. 44, N. ${ }^{\circ} 3,1954$, pp. 317-322.

HEILMAN, F. R. et al. - "Some laboratory and clinical observations on a new antibiotic, erythromycin (ilotycin)». In Proceedings of the Staff Meetings of the Mayo Clinic. V. 27, N. ${ }^{\circ} 15,1952$, pp. 285-304.

HINSHAW, H. C.; FELDMAN, W. H. - «Streptomycin in Treatment of Clinical Tuberculosis: A Preliminary Report". In Proceedings of the Staff Meetings of the Mayo Clinic. V. 20, 1945, pp. 313-318.

HOEFLE, Milton L. - «The early history of Parke-Davis and Compant». In Bulletin of the History of Chemistry. V. 25, N. ${ }^{\circ}$ 1, 2000, pp. 28-34.

JOHNSON, Balbina A.; ANKER, Herbert; MELENEY, Frank L. - «Bacitracin: A new antibiotic produced by a member of the B. subtilis group". In Science. V. 102, N. ${ }^{\circ} 2650,1945$, pp. 376-377.

KEEFER, Chester S. et al. - «Streptomycin in the treatment of infections - A Report of One Thousand Cases». In The Journal of the American Medical Association. V. 132, 1946, pp. 4-10; pp. 70-77.

LAWN, Stephen D.; ZUMLA, Alimuddin I. - «Tuberculosis». In The Lancet. V. 378, $\mathrm{N}^{\circ}$ 9785, 2011, pp.57-72.

LAX, Eric - The Mold in Dr. Florey's Coat. The story of the penicillin miracle. New York: Owl Books, 2005, pp. 251-252.

LEDNICER, Daniel - New drug discovery and development. New Jersey: John Wiley \& Sons, Inc, 2007, p. 2.

LODER, Bronwen; NEWTON, G. G. F.; ABRAHAM, E. P. - «The cephalosporin $\mathrm{C}$ nucleus (7-aminocephalosporanic acid) and some of its derivatives». In Biochemical Journal. V. 79, N. ${ }^{\circ} 4,1961$, pp. 408-416.

MAUROIS, André - The life of Sir Alexander Fleming. Oxford: The Alden Press, 1959, p. 162. 
MCGUIRE, J. M. et al. - «Ilotycin, a new antibiotic». In Schweizerische Medizinische Wochenschrift. V. 82, N. ${ }^{\circ} 41,1952$, pp. 1064-1065.

NEWTON, G. G. F. et al. - "Some observations on the biological properties of bacitracins A, B, and C». In British Journal of Pharmacology and Chemotherapy. V. 6, N. ${ }^{\circ}$ 3, 1951, pp. 417-429.

NEWTON, G. G. F.; ABRAhAM, E. P. - "Cephalosporin C, a new antibiotic containing sulphur and D- $\alpha$-aminoadipic acid». In Nature. V. 175, N. 4456 , 1955 , p. 548.

NEWTON, G. G. F.; ABRAHAM, E. P. - «Degradation, structure and some derivatives of cephalosporin N». In Biochemical Journal. V. 58, N. ${ }^{\circ} 1,1954$, pp. 103-111.

NEWTON, G. G. F.; ABRAHAM, E. P. - «Isolation of cephalosporin C, a penicillinlike antibiotic containing D-alpha-aminoadipic acid». In Biochemical Journal. V. 62, N. ${ }^{\circ} 4,1956$, pp. 651-658.

RENICHNICK, Pierre - "Os acidentes provocados pelos antibióticos». In Jornal do Médico. V. 21, N. ${ }^{\circ}$ 524, 1953, pp. 318-326.

RITCHIE, A. C.; SMITH, N.; FLOREY, H. W. - «Some biological properties of cephalosporin P1'. In British Journal of Pharmacology. V. 6, N. 3 3, 1951, pp. 430-444.

SCHATZ, A.; BUGLE, E.; WAKSMAN, S. A. - «Streptomycin, a Substance Exhibiting Antibiotic Activity Against Gram-Positive and Gram-Negative Bacteria». In Proceedings of the Society for Experimental Biology and Medicine. V. 55, 1944, pp. 66-69.

SOUZA, Marcus Vinícius Nora de; VASCONCELOS, Thatyana Rocha Alves «Fármacos no combate à tuberculose: passado, presente e futuro». In Quimica Nova. V. 28, N. ${ }^{\circ}$ 4, 2005, p. 679.

TOMASZ, Alexander - «Lessons from the first antibiotic era». In Fighting infection in the 21st Century. Oxford: Blackwell Science Ltd, 2000, pp. 198-216.

VIEIRA, Ismael Cerqueira - Conbecer, tratar e combater a 'peste branca'. A tisiologia e a luta contra a tuberculose em Portugal (1853-1975). Porto: Faculdade de Letras - Universidade do Porto, 2012, p. 268.

WAKSMAN, Selman A.; LECHEVALIER, Hubert A. - «Neomycin, a new antibiotic active against streptomycin-resistant bacteria, including tuberculosis organisms». In Science. V. 109, N. ${ }^{\circ} 2830,1949$, pp. 305-304.

WALLER, John - Fabulous science: fact and fiction in the bistory of scientific discovery. Oxford: Oxford University Press, 2004. p. 247.

WINTERNITZ, C. - «Fatal aplastic anemia following chloramphenicol (chloromycetin) therapy». In California medicine. V. 77, N. ${ }^{\circ}$, 1952, pp. 335-339. 